\title{
https://doi.org/10.48009/2_iis_2006_64-68 \\ INTEGRATED RESULTS REPORTING: MOVING TOWARD ELECTRONIC HEALTH RECORDS
}

\author{
Mary Helen Fagan, University of Texas at Tyler, mfagan@uttyler.edu \\ Carol Kilmon, University of Texas at Tyler, ckilmon@uttyler.edu \\ Tom Belt, University of Texas Health Center at Tyler, tom.belt@uthct.edu
}

\begin{abstract}
A key achievement marking the University of Texas Health Center at Tyler's movement toward Electronic Health Records (EHR) is the establishment of an integrated results reporting capability. Clinicians will be able to view results from most ancillary departments anywhere and at any time. This case study outlines the steps they took, the outcomes they achieved, and some of the lessons they have learned in the process.
\end{abstract}

Keywords: Electronic Health Records (EHR), Health Care, Hospitals, Case Study

\section{INTRODUCTION}

There is a long stream of research on the diffusion of innovations in the health care industry [1]. Research has found that 1) perceived attributes of the innovation, and 2) environmental, organizational, and leadership variables help to explain the progress of innovations in hospitals [6]. As the successful diffusion of information technology (IT) innovations has become increasingly important to the health care industry, more research has been conducted on the adoption of IT in health care settings. The research results generally indicate that "despite its potential to improve quality and reduce errors, use of information technology (IT) in the health care sector lags behind other sectors of the economy in the United States" [4].

Recently, the President of the United States, George Bush, has championed a push to build a national health information infrastructure, and, in particular, to provide an electronic health record (EHR) for every American [2]. These ambitious goals have highlighted the many challenges that the health industry and individual health care organizations face in coming up to speed with other industries in the information age. While some research has been done that specifically focuses on the adoption of EHR systems [7], this study is based upon the assumption that more insight into this particular IT innovation diffusion process is needed given its critical importance to health care delivery in the future.

\section{RESEARCH OBJECTIVE AND APPROACH}

Through the use of an in-depth case study, this research investigates some of the challenges and outcomes one particular organization, the University of Texas Health Center at Tyler (UTHCT), has faced in moving toward an EHR system for their enterprise. This paper provides a background of the UTHCT and then describes 1) the challenges they faced, 2) the process they employed in selecting and implementing a system, 3) the outcomes they have enjoyed, 4) the next steps they will take, and 5) the lessons they have learned.

Historically, research on the diffusion of innovations (DOI) has used a variance research approach. However, Everett Rogers, a prominent scholar in DOI has found "research on a topic such as the innovation-decision process should be quite different from the variance research that has predominated in the diffusion field" [8, p. 197]. Rogers recommends that process research, "a type of data gathering and analysis that seeks to determine the sequence of a set of events over time," be used instead [8, p. 196]. In line with this recommendation, this research study uses an in-depth, qualitative, case study approach to provide insight into the stages of the EHR adoption process at the UTHCT.

\section{BACKGROUND}

In 2000 the UTHCT was facing a serious challenge. They had implemented a beta version of a vendor's hospital information system (HIS) product and had encountered numerous system problems that could undermine the organization's future effectiveness. Therefore, in the spring of 2001, the UTHCT began to redefine their priorities for clinical informatics. The organization recognized that in today's changing healthcare environment, planning for information technology is a crucial ingredient in supporting the success of the business strategies and goals. As a result, the organization now is in the middle of a multi-phase, multi-year electronic health record (EHR) system implementation process. 
Experts have suggested that organizations go through a seven phase process of automation prior to successfully implementing a comprehensive EHR system, and that about $21 \%$ of hospitals have completed Stage 1, the installation of laboratory and radiology systems [5]. In March 2004, the UTHCT achieved this initial stage in the EHR implementation process and is now making progress in Stage 2, the development of a clinical data repository.

\section{ADDRESSING THE CHALLENGES}

The UTHCT is a multi-faceted organization located in east Texas that supports patient care, education and research. Over 138,500 outpatient visits and 3,700 inpatient stays each year are provided through their hospital, emergency care center, and more than twenty outpatient clinics. Some of the UTCHT educational offerings include a family practice residency program and three master degree programs. Finally, in addition to patient care and education, the UTCHT supports a wide range of research initiatives through the Center for Biomedical Research, the Center for Clinical Research and the Center for Pulmonary and Infectious Disease Control.

Faced with an unsatisfactory HIS, in 2001 the UTCHT embarked on a planning process with the goals of 1) placing core clinical data online, 2) reducing the reliance on paper medical records, and 3) preparing the organizational infrastructure and stakeholders for change. The planners envisioned an EHR system and clinical data repository that could help address the problems they had identified with the current paper medical record system. These issues included the fact that

- clinicians were sometimes unable to get the medical record on a timely basis for nonscheduled patient care and other non-scheduled medical record needs,

- it was often difficult to find clinical data within the medical record (e.g., due to large multiple volumes, disordered contents, untimely filing of reports),

- excessive amount of personnel and physical space were needed to maintain medical records,

- clinical personnel had a perceived need for "shadow charts,"

- it was difficult to comply with JCAHO with the current medical record,

- it was difficult to do research and clinical quality improvement with the current chart (e.g., to meet the need to search by patient type, diagnosis, test types, etc.),
- there was an increased risk of medical errors with the current medical record due to lack of timely availability, order and completeness of the medical record,

- it was difficult to do adequate clinical results review with the current record, and

- there was poor availability of clinical information for financial processes (coding, billing, etc.).

In summary, the results of the UTHCT needs assessment process concurred with other research that concludes that "the traditional paper record has become large, unmanageable, illegible, and frequently unavailable" [7, p. 7]. The desired new system would help address the problem by providing facility-wide access to core clinical data, logically displayed, when and where it was needed. Such online access would reduce the traditional reliance on paper charts, and, it was expected, greatly improve clinician's efficiency and effectiveness in performing daily tasks, resulting in improved patient care.

The UTHCT planners realized that that the anticipated information systems implementation would call for changes in process and organization and that key leadership must be willing to participate in and support the effort in order for it to succeed. To prepare the organizational infrastructure, they established the Health Information Management (HIM) department and consolidated functions, involved the Medical Information Management Committee (MIMC), and established a clinical HIS leadership group.

The planners also recognized the proposal would need widespread medical staff support to succeed. A key task was to get stakeholder input, especially from physicians, in order to facilitate acceptance. The right implementation pace, as one planner expressed it, would probably be too slow for technophiles, but, at the same time, too fast for technophobes. They expected that a gradual phase-in with training and pilot programs, along with a great deal of patience and flexibility as clinicians adjusted to work flow changes brought on by the new system, would help ensure success. Since researchers have found that $80 \%$ of the failures in the implementation of health care information systems stem from social and organizational factors, this emphasis on culture as well as technology at the UTHCT was well founded [3]. 


\section{SELECTING A VENDOR'S SYSTEM}

In early 2002, UTCHT put out a Request for Proposal (RFP) for selection assistance and selected a consulting group to assist in the HIS replacement selection process. A team of twenty five members was formed representing ancillary, registration, nursing, patient accounting, medical records, and information systems departments. The role of the consulting group was to provide a framework for the project manager and team to perform due diligence tasks as well as to educate the members of the Information Technology Executive Steering Committee (ITESC) on current HIS vendors and products.

The consultant provided a methodology for the vendor RFP selection process. They helped identify the basis for vendor inclusion in the selection process and helped the UTCHT utilize a variety of selection tools including reference calls, vendor demonstrations and site visits. The initial vendor selection process narrowed candidate vendors to six, which, after further analysis, was reduced to two potential system vendors. The consultant provided a needs assessment questionnaire describing physician access to the system, and sample patient encounter scenarios for inpatient and outpatient clinical order entry. After input from a large number of individuals spanning the entire organization, one vendor's system was selected.

The organization decided to take a phased approach to implementing their EHR system. In the first phase, twelve modules were purchased which would support clinical results viewing and transcription viewing within a single easy to navigate interface, along with system wide scheduling and hospital billing. By focusing on the implementation of an integrated results viewing system, they could expect to achieve a large number of benefits while minimizing the risks associated with a complex, organization wide, information systems implementation process.

\section{IMPLEMENTING THE INTEGRATED RESULTS REPORTING SYSTEM}

In early 2003, the UTCHT began the system installation process. It was the first time that the organization had implemented such a big information technology project. Approximately two thirds of the way into the project, they realized that external consultants were needed to help ensure the project goals were met. The UTHCT hired an external consultant who did a gap analysis to make sure all risks were covered. Based upon the gap analysis, the anticipated "go-live" date was delayed by six months. During implementation the consultants worked as integrated members of the implementation team. As a result, the installation turned out to be a good learning experience for the organization.

One of the challenging aspects of the system implementation involved transferring data from other systems and sources into the new system. The organization had contacted other users of the software and had heard that, for example, it was especially challenging to integrate cardiology results. However, the system interfaces turned out to be easier than anticipated. As various systems have been integrated into the new results reporting system, the organization has gained a more complete repository.

The ease of the "go live" process in March 2004 was a welcome relief for the organization after their bad experience with a HIS system that had never worked as anticipated. Key champions of the system implementation process noted how hard it can be to regain system acceptance once you lose it, especially with infrequent users. Project team members recognized that, based upon the previous experience with a system that did not meet expectations, there was some apprehension and skepticism about the implementation of the new system among potential users. However, the criticality of the process seemed to galvanize those who were responsible for the "golive" success. As one participant noted, "If you've had one failure, you better not fail again." In addition to the return on investment that was anticipated with the new system, the successful go-live experience produced numerous intangible benefits such as positive attitudes among users and confidence in the new integrated results reporting system. Their positive outcomes are reflected by the vendor's comments that the UTHCT installation has been one of the "best installs in the country."

\section{ASSESSING THE OUTCOMES}

The current results reporting system integrates data from feeder systems including imaging and therapeutic services, laboratory services, and pathology. The ability to access data through an easyto-use online interface represents a fundamental improvement in the accessibility of health care information in the organization and helps improve the efficiency and effectiveness of clinical care.

Physician acceptance of the system has been good and data that has been gathered since implementation shows increasing usage. It was a quantum leap for physicians to go from a system where someone 
retrieved laboratory data for them to a system where they looked up results themselves on the computer. The system implementation team attributes the fact that there has been relatively little physician resistance to 1) the intensive involvement of physicians in product evaluation, 2) their use of oneon-one just-in-time training, and 3) the presence of strong physician champions.

A variety of features support the clinician in performing their job. For example, the results reporting system will indicate if there are new unretrieved results for a patient, and order tracking helps ensure that the physician sees the results. When the results are retrieved, abnormal results are highlighted in distinct colors, and clinical decision making is supported by the ability to easily generate a number of graphical displays of the data. For example, clinicians have the ability to graph three data items on either layered or separate stacked graphs, with up to 128 measurements on one graph. A sample graph might display the values and ranges for the white blood count, red blood count, and platelet count on one screen, each represented by a different color. The laboratory and blood bank system modules send the normal ranges for the various data items for use in generating the graph.

Furthermore, the system allows information to be aggregated into disease specific panels. For example, if a patient had pneumonia, the system would draw together all the lab information (chest x-ray, medications, etc.) that are relevant to this diagnosis and show them in one place. This constitutes a major improvement over the paper based patient chart where a physician would be required to flip from section to section to gain the information (assuming that the chart was up-to-date and complete). This system feature reduces the number of "clicks" that it takes for a physician to retrieve the information from various screens and provides a desirable "flattening" of the system record.

\section{TAKING THE NEXT STEPS}

A comprehensive clinical data repository is being built at the UTCHT that provides additional capabilities beyond supporting improved clinical care. For example, the UTHCT expects to be able to improve the efficiency of business functions/operations through the analysis of this data repository. In addition, this clinical data repository will improve their ability to perform patient research.

As a prerequisite to the next phase, electronic transcription will be implemented. The system will be upgraded to have basic voice recognition functionality for physician dictation. The planned implementation will conduct back-end transcription, and then this information will be "cleaned up" by hand. A key requirement for this system is that it will be a learning system, learning as it goes to more accurately transcribe physician dictation.

The next steps toward building a more complete EHR will require process change and therefore are anticipated to be even more challenging. One of the most difficult parts is expected to come when physician orders and computerized medication prescribing are implemented. However, the planners are looking forward to automating the workflow and seeing the anticipated benefits accrue from this effort.

Currently, a pilot project is providing physicians with a tablet PC to allow them access to the integrated results reporting system. Since this trial of handheld devices is limited to the results reporting system, its usefulness is limited by the fact that clinicians must still possess the paper chart. However, the preliminary results are encouraging: physicians are coming to rely upon the convenience and mobility of handheld devices for results information, as well as for clinical references. It is likely that as the technology converges further so that the physician's phone, beeper, and wireless computing tablet can be merged into one handheld device, acceptance of this innovation will take off.

In the future, system implementers are looking forward to more comprehensive decision support systems (DSS). For example, they anticipate providing a context sensitive DSS that will deliver specific information on a patient's condition (e.g., hypertension) and the most applicable evidence based practices without the user having to follow various links to retrieve the information from diverse sources. They also look forward to DSS applications that will be tailored to the specific situation and that take into account pertinent patient information. For instance, in the area of medication safety, a DSS will review information about patient's allergies and current medications, and provide drug interaction alarms if a contraindicated medicine (e.g., penicillin) is prescribed.

In addition, the system planners are considering the implementation of a patient portal using a "bolt-on" software package that will interface with their current system. This proposed patient portal will allow patients to access test results, schedule appointments, request medication refills, and communicate via email with providers. The portal application would 
alert the provider if a patient has not logged on to access their results. Because the majority of their phone calls concern prescription refill requests, such a system should improve operational efficiency as well as patient satisfaction with services rendered. However, the planners are cognizant of many issues relating to patient portal implementation that must be addressed. The issues are not technical; instead, they are concerned with training up their patient population to use such tools and with various new legal issues which relate to these new practices.

\section{LESSONS LEARNED}

In successfully implementing the integrated results reporting system, the UTHCT has taken an important first step towards the eventual implementation of a comprehensive EHR system. Some suggestions, based upon their experience, that might help others following along this path include the following:

- Spend a lot of time planning and understanding what you want to achieve. Technology is not the secret - there are a number of good choices. The key is to know what you want.

- Do your homework. Use consultants as needed. Other providers who have done similar projects are valuable sources of information and advice. Have a strong network. Don't make the same mistake as others.

- Have good leadership and remain true to your objectives. Once you are into the project, it is easy to be diverted by the number of software options available and lose sight of you original goals.

- Be self-critical about the planning and implementation process. Make sure you have adequate resources (especially human resources).

- Be flexible and willing to reconsider the original plan during implementation. Don't be afraid to make changes if needed.

As the organization proceeds with the rollout of additional EHR functionality, these principles should continue to be put into practice, and, it is hoped, help ensure their ongoing implementation success.

\section{REFERENCES}

1. Berwick, D. M. (April 16, 2003). Disseminating Innovations in Health Care, JAMA, 289(15), 1969-1975.

2. Brailer, D. (2004). The Decade of Health Information Technology: Delivering Consumercentric and Information-rich Health Care. Report to the Secretary of the Department of Health and Human Services, July 2004, http://www.hhs.gov/onchit/framework/hitframew ork.pdf.

3. Burley, L. \& Scheepers, H. (2002). From HealthCare Professional to System Developer: Emerging Trends in Mobile Technology Development, in Seventh CollECTeR Conference on Electronic Commerce (Eds, Cooper, J., Burgess, L., Alcock, C. and Win, K. T.) University of Wollongong, Melbourne, Australia.

4. Burt, C. W. \& Hing, E. (2005). Use of Computerized Clinical Support Systems in Medical Settings: United States, 2001-03, Advance Data From Vital and Health Statistics, No. 353, March, 15, 2005, U.S. Department of Health and Human Services.

5. Health Data Management. Long, Winding Road Ahead for RHIOs, Health Data Management, October 12, 2005, http://www.healthdatamanagement.com.

6. Meyes, A. D. \& Goes, J. B. (1988). Organizational Assimilation of Innovations: A Multi-level Contextual Analysis, Academy of Management Journal, 31(4), 897-923.

7. Murphy, G. F. \& Waters, K. A., (1999). EHR Vision, Definition, and Characteristics, in Electronic Health Records: Changing the Vision, eds. Murphy, G. F., Hanken, M.A., and Waters, K. A., W.B. Saunders Company.

8. Rogers, E. M., (2003). Diffusion of Innovations, Free Press. 